\title{
Adherence to oral anticoagulant therapy in secondary stroke prevention - impact of the novel oral anticoagulants
}

This article was published in the following Dove Press journal:

Patient Preference and Adherence

23 November 2015

Number of times this article has been viewed

\section{Sebastian Luger' \\ Carina Hohmann² \\ Daniela Niemann' \\ Peter $\mathrm{Kraft}^{3}$ \\ Ignaz Gunreben ${ }^{3}$ \\ Tobias Neumann-Haefelin ${ }^{2}$ \\ Christoph Kleinschnitz ${ }^{3}$ \\ Helmuth Steinmetz' \\ Christian Foerch' \\ Waltraud Pfeilschifter ${ }^{1}$ \\ 'Department of Neurology, University Hospital Frankfurt, Frankfurt am Main, ${ }^{2}$ Department of Neurology, Klinikum Fulda gAG, Fulda, ${ }^{3}$ Department of Neurology, University Hospital Würzburg, Würzburg, Germany}

Correspondence: Waltraud Pfeilschifter Department of Neurology, University Hospital Frankfurt, Theodor-Stern-Kai 7, 60590 Frankfurt am Main, Germany

Tel +496963016395

Fax +4969630I 4498

Email w.pfeilschifter@med.uni-frankfurt.de
Background: Oral anticoagulant therapy (OAT) potently prevents strokes in patients with atrial fibrillation. Vitamin K antagonists (VKA) have been the standard of care for long-term OAT for decades, but non-VKA oral anticoagulants (NOAC) have recently been approved for this indication, and raised many questions, among them their influence on medication adherence. We assessed adherence to VKA and NOAC in secondary stroke prevention.

Methods: All patients treated from October 2011 to September 2012 for ischemic stroke or transient ischemic attack with a subsequent indication for OAT, at three academic hospitals were entered into a prospective registry, and baseline data and antithrombotic treatment at discharge were recorded. At the 1-year follow-up, we assessed the adherence to different OAT strategies and patients' adherence to their respective OAT. We noted OAT changes, reasons to change treatment, and factors that influence persistence to the prescribed OAT.

Results: In patients discharged on OAT, we achieved a fatality corrected response rate of $73.3 \%(n=209)$. A total of $92 \%$ of these patients received OAT at the 1-year follow-up. We observed good adherence to both VKA and NOAC (VKA, 80.9\%; NOAC, 74.8\%; $P=0.243$ ) with a statistically nonsignificant tendency toward a weaker adherence to dabigatran. Disability at 1-year follow-up was an independent predictor of lower adherence to any OAT after multivariate analysis, whereas the choice of OAT did not have a relevant influence.

Conclusion: One-year adherence to OAT after stroke is strong $(>90 \%)$ and patients who switch therapy most commonly switch toward another OAT. The 1-year adherence rates to VKA and NOAC in secondary stroke prevention do not differ significantly between both therapeutic strategies.

Keywords: stroke, prevention, vitamin $\mathrm{K}$ antagonists, non-VKA oral anticoagulants, adherence

\section{Introduction}

Oral anticoagulant therapy (OAT) is an effective treatment to prevent strokes in patients with atrial fibrillation (AF). ${ }^{1,2}$ As for any long-term therapy, an important prerequisite for its efficacy is the patient's faithfulness to regular medication intake. Especially in chronic conditions, adherence is often astonishingly low, with adherence rates around $50 \%$ after 1 year. $^{3}$

For decades, vitamin $\mathrm{K}$ antagonists (VKA) were the only option for long-term OAT. Adherence to OAT for the prevention of stroke in AF patients seems to be somewhat stronger but is still problematic considering that stroke prevention is a vital indication. A recent report from a large insurance-based US cohort in 2010 showed that more than $25 \%$ of patients prescribed warfarin for stroke prevention discontinued this treatment within the 1 st year despite a low rate of hemorrhagic complications. ${ }^{4}$ 
Medication adherence is supported by factors such as higher levels of education, a stable social background, feeling wellinformed about the therapy and a trustful patient-physician relationship, but also the severity of the illness positively influences medication adherence. ${ }^{5}$ Therefore, a stroke as a dramatic experience could reinforce adherence to OAT as a secondary prevention strategy. On the other hand, neuropsychiatric issues such as post-stroke depression and dementia could impair adherence and the delegation of medication adherence to a caregiver due to physical impairment has to be taken into consideration.

In the last few years, alternatives to warfarin and other VKAs have become available. The novel non-VKA oral anticoagulants (NOAC) targeting single coagulation factors (dabigatran - factor II, rivaroxaban, apixaban, edoxaban factor $\mathrm{Xa}$ ) have shown at least non-inferior risk-to-benefit profiles in randomized controlled clinical trials. ${ }^{6-9}$ Especially intracranial hemorrhage, the most feared complication of long-term anticoagulation, occurred less frequently in the NOAC arms of all aforementioned randomized controlled clinical trials. ${ }^{10}$ The advent of the novel NOAC has triggered many controversial debates. Especially the question whether NOAC may facilitate or endanger patients' long-term medication adherence is yet unanswered.

The need for regular coagulation checks, which provide a constant feedback on medication intake, is often cited as a factor that might strengthen adherence to VKA. ${ }^{5}$ But it has also been reported that medication-associated anxiety is common among VKA-treated patients ${ }^{11}$ and the stress of regular blood tests may weigh on their medication adherence. Along this line, the superior risk-to-benefit ratio of the novel NOAC might reduce fears linked to OAT and enhance medication adherence.

We prospectively assessed and compared 1-year adherence to OAT for VKA and NOAC in the secondary prevention of stroke in a reasonably large cohort of consecutive patients from three large academic stroke centers.

\section{Patients and methods}

We prospectively collected data on 1-year medication adherence to OAT and self-reported medication adherence for secondary stroke prevention in a registry that was run by three large academic stroke centers in Germany: Frankfurt University Hospital, Würzburg University Hospital, and Marburg University Hospital (Klinikum Fulda) (inclusion of patients from October 2011 to September 2012, end of follow-up October 2013). Dabigatran was marketed in Europe for stroke prevention in AF in September 2011. Rivaroxaban obtained approval for this indication on the EU market in December 2011. The registry was approved by the ethics committees of the Frankfurt University Hospital, Würzburg University Hospital, and Marburg University Hospital (Klinikum Fulda). Written informed consent was obtained from all patients/ next-of-kin who returned the questionnaire. Verbal informed consent was obtained from patients/next-of-kin participating in the telephone interview. We noted that in these cases, there often were obstacles to mailing the written consent form and questionnaire, such as impaired mobility. Therefore, we abstained from written consent and the interviewer documented the verbal consent in written form. This consent procedure was approved by the ethics committees.

We identified all patients with the discharge diagnoses of ischemic stroke (ICD-10 code I63) or transient ischemic attack (G45) in combination with either AF detected during the hospital stay or a documented history of AF (I48), who were discharged from our stroke services from October 2011 to September 2012 $(n=596)$. Stroke did not have to be of cardioembolic origin. With a $\mathrm{CHA}_{2} \mathrm{DS}_{2}-\mathrm{VASc}$ score $\geq 2$, all these patients had an indication for OAT. ${ }^{12}$ We recorded age, sex, presence of hypertension and diabetes, National Institutes of Health Stroke Scale score at admission, modified Rankin Scale (mRS) score, and antithrombotic treatment at discharge. As a noninterventional study, this registry did not interfere with physicians' choices of OAT for secondary stroke prevention. Patient- and physician-related factors that influenced the choice of OAT have been published in a previous analysis from this registry. ${ }^{13}$

At follow-up after 1 year, all patients were sent a follow-up letter including a questionnaire addressing their current antithrombotic treatment, potential changes of therapy, and negative side effects/adverse events in the outpatient setting. We opted for a patient-centered view of adherence. Patients were accounted as adherent to the OAT installed at discharge if they reported to be taking this drug and as adherent to any OAT if they reported taking any OAT at 1-year follow-up. Additionally, the eight-point Morisky Medication Adherence Scale (MMAS-8), ${ }^{14,15}$ a self-report measure of medication adherence within the last 2 weeks, which has been reported to correlate well with pharmacy fill rates, ${ }^{16}$ was used to assess adherence to the current OAT regimen. Adherence was graded on a three-item scale of high adherence ( 8 points on the MMAS), medium adherence (6-7 points), and low adherence ( $0-5$ points). Functional neurological status at 1-year follow-up was recorded by means of the self-reported mRS. If patients did not return the questionnaire, we contacted them or their next-of-kin, who had been recorded during the hospital stay, by telephone. 
We recorded the choice of OAT installed at discharge and the OAT reported by the patient at 1-year follow-up and noted the respective shares of VKA and NOAC. The present analysis is focused on adherence to OAT as a secondary prevention strategy after stroke. Therefore, we selected all patients who had an OAT installed at discharge from our stroke units $(\mathrm{n}=324)$ for all further analyses. Our main outcome parameters were 1-year adherence to the specific choice of OAT that was installed at discharge from our stroke units. We also noted adherence to any OAT. We assessed self-reported medication adherence, factors influencing adherence, OAT switches, patient-reported reasons for changes of OAT regimens, and negative side effects and adverse events as conveyed by the patients.

We used IBM SPSS, Version 20 (IBM Corporation, Armonk, NY, USA) for data analysis and graph presentation. By means of a binominal test statistics, we compared the prescription rate between VKA and NOAC, both at hospital discharge and at 1-year follow-up. Statistical significance of between group differences of: 1) patients discharged on VKA as compared to patients discharged on NOAC and 2) patients adherent to any OAT as compared to patients who reported non-adherence or who were lost to follow-up were tested with Student's $t$-test for parametric data following a normal distribution, and with chi-square test for nonparametric data. Bonferroni correction was used to adjust for multiple testing. We used a binary logistic regression model with multivariate adjustment to define factors that independently influence adherence to OAT after stroke, adjusting for age, sex, mRS at discharge, and at 1-year follow-up and choice of OAT at discharge and indicated odds ratio, $95 \%$ confidence interval, and $P$-values.

\section{Results}

\section{Response rate and survival}

Of the 596 patients entered into the registry, 324 (54.4\%) had an OAT installed at discharge from our stroke units. The median length of stay was 7 days, $25 \%-75 \%$ interquartile range 5-11 days. These patients were followed up as a secondary prevention cohort (Figure 1). At 1-year follow-up, 159 of these patients or their next-of-kin (49.1\%) returned the questionnaire by mail. In 11 cases $(3.4 \%)$, we received the written information from patients' next-of-kin that the patient had died and in an additional 21 cases (6.5\%), the letter was returned with the postal annotation "recipient reported deceased". In all other cases, we attempted to contact the patient and/or next-of-kin for a telephone interview. We conducted telephone interviews with 61 patients or next-of-kin (18.8\%) and were informed orally by the patients' next-of-kin that the patient had died during the follow-up period in seven cases $(2.2 \%)$, leading to a cumulative 1-year mortality rate of $12.0 \%$. Seventy-six patients $(23.5 \%)$ were lost to follow-up, which amounts to a fatality corrected response rate of $73.3 \%$ (absolute response rate $64.5 \%$ ). The mRS score distribution at 1-year follow-up showed that although we received information from patients with varying degrees of disability, the majority of patients who participated in the survey were fairly independent with only $20.5 \%$ reporting an $\mathrm{mRS} \geq 3$ (Figure 2 ) and $32.7 \%$ reporting to receive nursing care of any kind at home or in an institution. There was a significant difference in the proportion of patients depending on help in the activities of daily living ( $\mathrm{mRS}>2$ ) between patients spontaneously returning the written questionnaire and patients/families participating in the telephone interview ( $15.8 \%$ vs $34.4 \%, P=0.01$ ).

\section{Anticoagulation rates at discharge}

At discharge from our stroke units, the prescription rate was balanced between VKA and NOAC ( $48.5 \%$ vs $51.5 \%$ of all anticoagulated patients, binominal test statistics $P=0.579$ ). The overall OAT rates at discharge from stroke centers were balanced between the three participating hospitals (Frankfurt, 55.6\%; Würzburg, 54.9\%; and Fulda, 53.5\%). However, we found significant heterogeneity of the percentage of NOAC among OAT prescriptions between the two university hospitals Frankfurt (60.0\%) and Würzburg (67.3\%) on the one side and the non university tertiary stroke center Fulda on the other side (33.8\%, $P<0.001$ for comparisons with Frankfurt and Würzburg).

\section{No relevant demographic differences between patient groups discharged on VKA or NOAC}

There were no significant differences in age, sex distribution, prevalence of the cardiovascular risk factors hypertension and diabetes mellitus, rate of prior independence, stroke severity, and disability at discharge between the group prescribed VKA and the group that received NOAC (Table 1). Consistent with a previous analysis from our group, ${ }^{13}$ younger age was a strong but yet nonsignificant factor to prompt stroke neurologists to prescribe NOAC instead of VKA. The demographic characteristics by specific choice of OAT are detailed in Table S1.

\section{Anticoagulation rates at I-year follow-up}

Information on adherence to OAT in the outpatient setting at 1-year follow-up is given in Table 2 according to the specific 


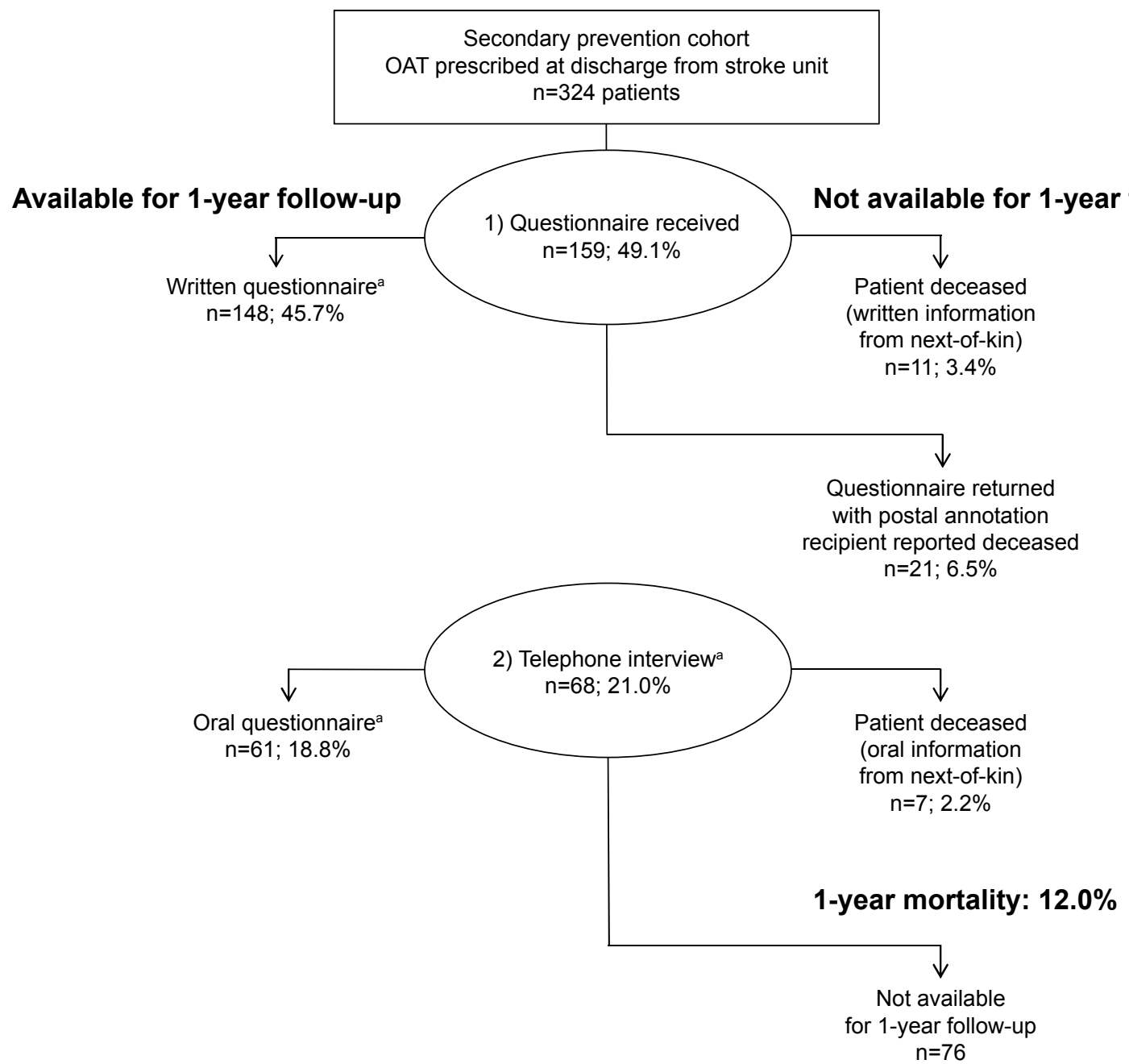

1-year follow-up cohort $n=209 ; 64.5 \%$

fatality corrected

response rate: $73.3 \%$

Lost to follow-up: $23.5 \%$

Figure I Flow chart illustrating the participation of patients who had an OAT installed at discharge from the stroke unit after stroke or transient ischemic attack. Notes: ${ }^{a}$ By patient or next-of-kin. The rates indicated as \% refer to the initial secondary prevention cohort of $n=324$ patients. To obtain the fatality corrected response rate, we calculated the ratio between completed questionnaires and the number of the entire secondary prevention cohort ( $\mathrm{n}=324$ ) minus the patients that were reported as deceased at I year $(n=39)$.

Abbreviation: OAT, oral anticoagulant therapy.

choice of OAT that was installed at discharge from our stroke units (VKA, dabigatran, rivaroxaban). The percentage of patients lost to follow-up did not differ significantly between groups (patient group discharged on VKA, 25.5\%; dabigatran, 25.9\%; and rivaroxaban, 23.2\%).

Adherence to the OAT regimen that was installed at discharge was generally acceptable for all three therapies at 1-year follow-up (VKA, 80.9\%; dabigatran, 70.9\%; and rivaroxaban, $83.3 \%$ ) with a relevant but nonsignificant tendency toward a lower adherence to dabigatran. Comparing VKA with both NOAC grouped together, adherence did not differ significantly between both OAT strategies (VKA, $80.9 \%$; NOAC, $74.8 \%$; $P=0.243$ ). By contrast, adherence to the general principle of any OAT (independent of the specific drug) was very good with $92.8 \%$ of all patients available for follow-up.

Of all three groups, the patients discharged on dabigatran showed the highest switching rate to other antithrombotic therapies $(28.9 \%)$ as compared to patients receiving VKA (16.4\%) and rivaroxaban (11.4\%). Most patients who switched therapy were prescribed another OAT, and only $5 \%-10 \%$ of patients in each group discontinued OAT in 


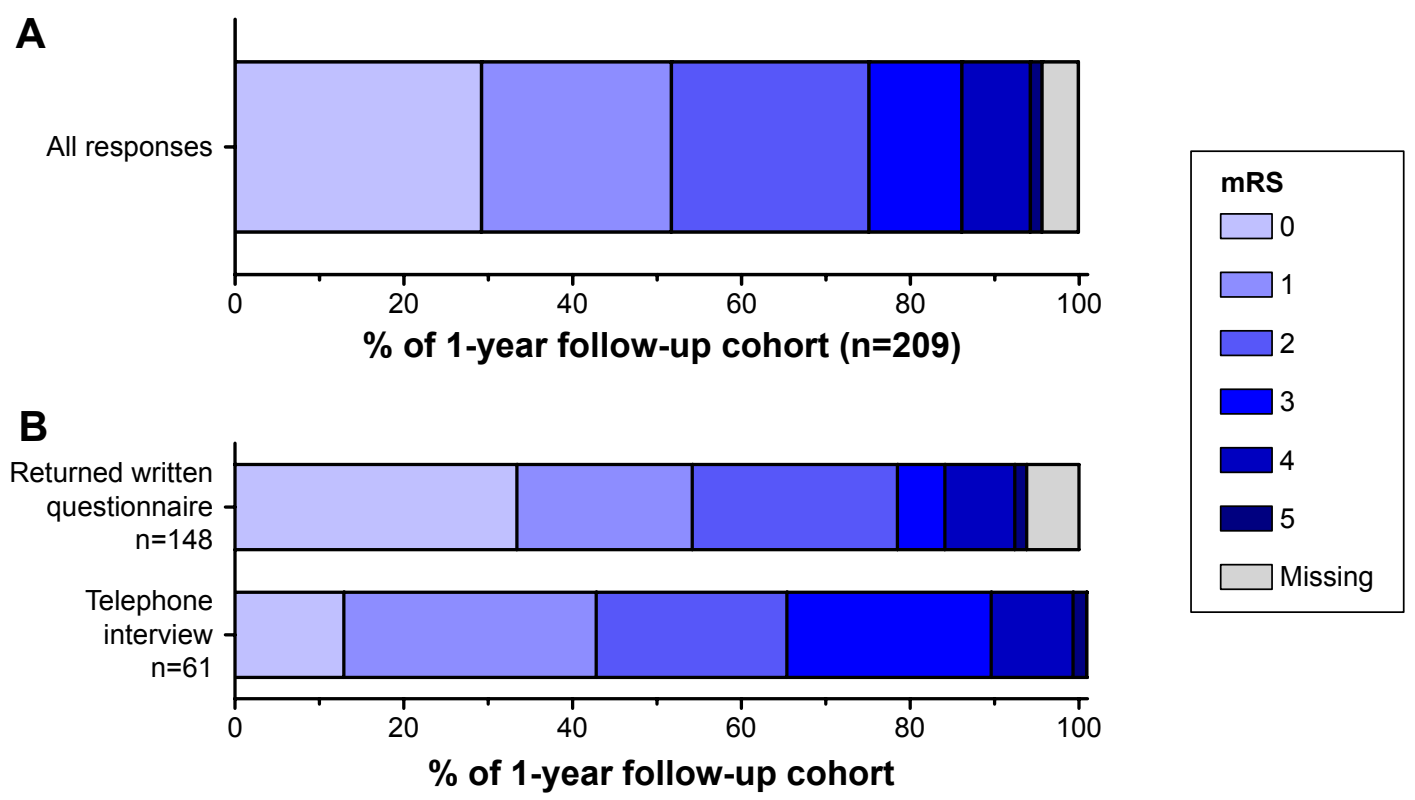

Figure 2 Global neurological function of the patients at I-year follow-up.

Note: Graded on the modified Rankin Scale score (mRS) according to the written or oral answers of patients and/or their next-of-kin. (A) All participating patients, (B) by mode of participation.

general during the 1-year follow-up. Patients' self-reported day-to-day medication adherence during the last 2 weeks as expressed on the MMAS- 8 correlated well with the report to be on treatment with the specific OAT that had been prescribed at discharge. A total of $81.0 \%$ of patients taking VKA reported high adherence as compared to $84.8 \%$ of patients discharged on rivaroxaban and $70.3 \%$ of patients discharged on dabigatran (Figure 3, differences not significant). Comparing VKA with both NOAC grouped together, medication adherence did not differ significantly between both OAT strategies (VKA, 83.5\%; NOAC, 75.8\%; $P=0.153$ ).

Mortality at 1 -year follow-up was significantly lower in the group of patients discharged on dabigatran (3.6\%) in comparison with the other two OAT groups (VKA, 17.2\%; $P<0.001$ and rivaroxaban, $14.3 \% ; P=0.020$, significant after Bonferroni adjustment). Characterizing the patient cohort by specific choice of OAT, we noted that the patient cohort prescribed dabigatran was on average younger, had less comorbidities and a higher rate of prior independence, but these differences did not reach statistical significance in our patient sample (Table S1). Patients on dabigatran had a statistically significant lower rate of prior VKA use compared to patients prescribed rivaroxaban $(11.7 \%$ vs $30.4 \%$, $P=0.022)$ and patients prescribed VKA $(64.3 \%, P<0.001$, both significant after Bonferroni adjustment).

\section{Reasons to switch OAT}

Among the three OAT regimens, dabigatran showed a considerably higher switching rate $(28.9 \%)$ as compared to VKA (16.4\%) and rivaroxaban (11.4\%, Table S2). According to patient-reported reasons for treatment change, this was most often due to gastrointestinal side effects, a worsening of renal function during the follow-up period and a presumed easier use of a once daily (od) dosing regimen. Among patients who were started on VKA at discharge from our stroke units, labile international normalized ratio (INR)

Table I Demographic characterization of patients' anticoagulated with VKA or NOAC at discharge from our stroke units

\begin{tabular}{|c|c|c|c|}
\hline & VKA at discharge $(n=157)$ & NOAC at discharge $(n=167)$ & P-value \\
\hline Age (years) & $77(73-83)$ & $76(7 \mid-82)$ & 0.084 \\
\hline \multicolumn{4}{|l|}{ Mean, $25 \%-75 \%$ interquartile range } \\
\hline Female sex & $49.0 \%$ & $51.8 \%$ & 0.658 \\
\hline Hypertension & $94.9 \%$ & $93.5 \%$ & 0.641 \\
\hline Diabetes mellitus & $28.7 \%$ & $23.2 \%$ & 0.652 \\
\hline Prior independence & $79.6 \%$ & $81.5 \%$ & 0.299 \\
\hline NIHSS (median, $25 \%-75 \%$ interquartile range) & $2(I-5)$ & $3(I-6)$ & 0.191 \\
\hline mRS at discharge (median, $25 \%-75 \%$ interquartile range) & I (0-2) & I (0-2) & 0.928 \\
\hline
\end{tabular}

Abbreviations: mRS, modified Ranking Scale score; NIHSS, National Institutes of Health Stroke Scale; NOAC, non-VKA oral anticoagulants; VKA, vitamin K antagonists. 
Table 2 Persistence to OAT at I year by specific choice of OAT at discharge

\begin{tabular}{|c|c|c|c|}
\hline & VKA $(n=157)$ & Dabigatran $(n=|| 2)$ & Rivaroxaban $(n=56)$ \\
\hline Patient deceased at I-year follow-up & $17.2 \%(n=27)$ & $3.6 \%(n=4)$ & $14.3 \%(n=8)$ \\
\hline Patient lost to follow-up & $22.9 \%(n=36)$ & $25.9 \%(n=29)$ & $20.0 \%(n=I I)$ \\
\hline Patients available for follow-up & $60.0 \%(n=94)$ & $70.5 \%(n=79)$ & $66.1 \%(n=37)$ \\
\hline VKA at follow-up & $80.9 \% *$ & $8.9 \% *$ & $11.1 \% *$ \\
\hline Dabigatran at follow-up & $5.3 \% *$ & $70.9 \% *$ & $0 \% *$ \\
\hline Rivaroxaban at follow-up & $7.4 \% *$ & $11.9 \% *$ & $83.3 \% *$ \\
\hline Antiplatelets & $0 \% *$ & $8.9 \% *$ & $0 \% *$ \\
\hline No answer/ & $4.3 \% *$ & $0 \% *$ & $2.8 \% *$ \\
\hline \multicolumn{4}{|l|}{ Other antithrombotic } \\
\hline No antithrombotic therapy & $0 \% *$ & $0 \% *$ & $2.8 \% *$ \\
\hline Adherence to any OAT & $93.6 \% *$ & $91.2 \% *$ & $94.4 \% *$ \\
\hline
\end{tabular}

Notes: Bold fields mark the adherence to the OAT installed at discharge and the adherence to any OAT, both at I-year follow-up. *Percentage of patients available at I-year follow-up.

Abbreviations: OAT, oral anticoagulant therapy; VKA, vitamin $\mathrm{K}$ antagonists.

values were the most common reason to switch from VKA to a NOAC. Interestingly, neither patients' concerns about VKA or NOAC nor the costs of therapy seemed to play a very important role in the decision to switch OAT during the observation period.

\section{Factors influencing adherence to OAT after stroke}

To identify factors that impede patients' adherence to OAT after stroke, we compared the group of adherent patients $(n=194)$ to the group of patients who reported to be nonadherent at follow-up $(n=15)$. In a univariate analysis, the adherent group showed tendencies to be younger, with a lower disability score (mRS) and a higher degree of independence at 1-year follow-up (Table 3). The prescription frequencies of VKA and NOAC did not differ between the adherent and the non-adherent group. Multivariate analysis confirmed the degree of disability (mRS) at 1-year follow-up as an independent predictor of lower adherence (Figure 4).

\section{Discussion}

In this prospective registry assessing 1-year adherence with OAT in the secondary prevention of stroke with a special focus on the impact of the novel NOAC, we found acceptable adherence rates for all three substances (VKA, $81 \%$; dabigatran, $71 \%$; and rivaroxaban, $83 \%$ ) at 1 -year follow-up but excellent adherence to the general principle of OAT for any of the three substances (92\%). Patients who switched treatment most commonly switched to another OAT. The reasons for switching OAT during the 1-year observation period reflect the known disadvantages of each drug. Thus, our findings underline the assumption that the new diversity of drugs for long-term OAT will lead to the

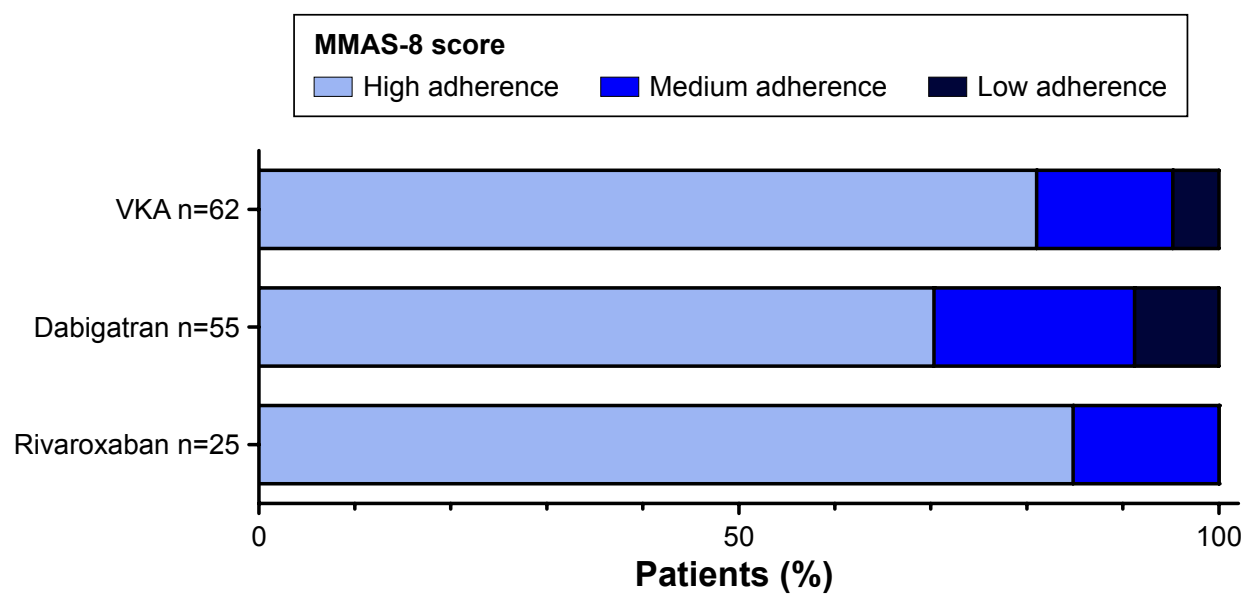

Figure 3 Patients' self-reported adherence assessed with the MMAS-8.

Notes: Only the patients who had returned the written questionnaire and had filled out the Morisky score at the end of the I-year follow-up period ( $\mathrm{n}=\mathrm{I} 42$ ) were included in the analysis, excluding patients or next-of-kin who provided their information via telephone interview.

Abbreviations: MMAS-8, eight-point Morisky Medication Adherence Scale; VKA, vitamin K antagonists. 
Table 3 Univariate comparison of patient characteristics between persistent and nonpersistent patients

\begin{tabular}{llll}
\hline & Adherent (n=194) & Non-adherent $(\mathbf{n}=\mathbf{I 5})$ & $\boldsymbol{P}$-value \\
\hline Age (year) mean, 25\%-75\% interquartile range & $75(7 \mathrm{I}-8 \mathrm{I})$ & $76(73-86)$ & $0.2 \mathrm{I4}$ \\
Female sex & $45.4 \%$ & $40.0 \%$ & $0.79 \mathrm{I}$ \\
mRS at discharge (median, 25\%-75\% interquartile range) & $\mathrm{I}(0-2)$ & $\mathrm{I}(0-2)$ & 0.616 \\
mRS at I year (median, 25\%-75\% interquartile range) & $\mathrm{I}(0-2)$ & $2(\mathrm{I}-3)$ & 0.184 \\
Independence at follow-up & $70.4 \%$ & $36.4 \%$ & 0.054 \\
VKA prescription & $45.4 \%$ & $40.0 \%$ & $0.79 \mathrm{I}$ \\
NOAC prescription & $54.6 \%$ & $60.0 \%$ & $0.79 \mathrm{I}$ \\
\hline
\end{tabular}

Note: ${ }^{\circledR}$ At discharge from the stroke units.

Abbreviations: mRS, modified Ranking Scale score; NOAC, non-VKA oral anticoagulants; VKA, vitamin K antagonists.

often called-for broader use of OAT in patients with AF by enabling physicians to tailor the treatment to the needs of the individual patient. In contrast, our study does not support the widespread hypothesis that the lack of regular "adherence checks" via mandatory coagulation monitoring might lead to lower adherence to NOAC.

We identified disability at 1-year follow-up, expressed on the mRS and by self-reported need of nursing care 1 year after stroke, as an independent predictor of non-adherence. This is an interesting finding and at this point, our data do not allow to answer the question whether this is mainly rooted in disability (patient only has limited cognitive and physical resources to hold up adherence or OAT is deemed dangerous in case of frequent falls) or futility (patient's and physician's attitude that secondary prevention is no longer worth the effort of daily medication intake due to a low quality of life).

We therefore used two alternative strategies in parallel to assess medication adherence. The questionnaire at 1-year follow-up evaluated the current treatment at that time point (persistence) as detailed by the patient. In parallel, patients completed the MMAS-8, a self-report instrument to measure day-to-day adherence. We found a very high correlation between the results of both assessment strategies. The MMAS- 8 in turn has been validated to correlate well with prescription refill rates. ${ }^{16}$ Beyond that, in patients taking warfarin, high adherence scores on the MMAS-8 corresponded to a longer time in the therapeutic INR range. ${ }^{17}$

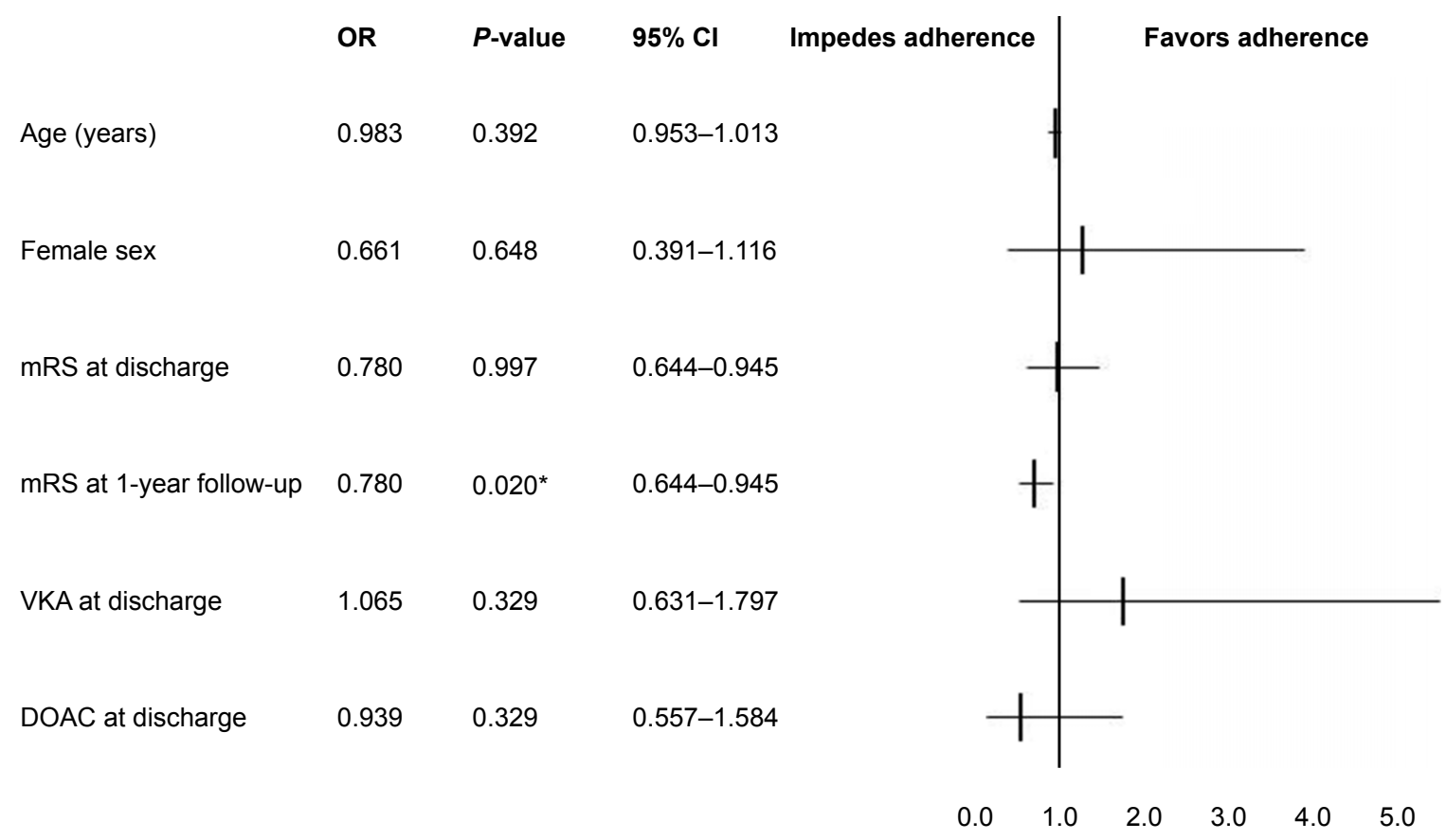

Figure 4 Multivariate analysis of factors influencing adherence to OAT in stroke patients.

Notes: We analyzed all patients with available written or oral questionnaire $(n=209)$ and adjusted for age, sex, mRS at discharge, and at I-year follow-up and choice of OAT at discharge. $* P<0.05$.

Abbreviations: $\mathrm{Cl}$, confidence interval; DOAC, direct oral anticoagulants; mRS, modified Ranking Scale score; OAT, oral anticoagulant therapy; OR, odds ratio; VKA, vitamin $\mathrm{K}$ antagonists. 
Stroke survivors are a challenging population for follow-up evaluations because of their advanced age (mean age $>75$ years in this registry) and disability, factors that often impair communication and mobility. The relatively small sample size of 209 patients available for 1-year follow-up clearly is a limitation of our study. It is noteworthy that patients spontaneously returning the written questionnaire were significantly less often affected by disabilities than patients who did not return the questionnaire but were available for a telephone interview. Even in comparison to age-matched populations, stroke patients show a higher mortality. This may explain the relatively low absolute response rate of $64.5 \%$. Corrected for fatalities reported at 1-year follow-up, the response rate reached $73.3 \%$. Since stroke recurrence and death rates are higher in $\mathrm{AF}$ patients not receiving OAT, it can be speculated that a relevant proportion of the patients who died during the observation period had not been adherent to OAT. However, it should be considered that the patients who died were significantly older at discharge than those available for 1-year follow-up (mean age $80.0 \pm 10.9$ vs $75.6 \pm 8.2$ years, $P<0.05$ ). Therefore, a proportion of deaths might have been unrelated to the use or nonuse of OAT. Most importantly, our main aim was to evaluate the impact of the novel NOAC on medication adherence in stroke survivors requiring OAT. Therefore, it is important to note that the proportion of patients lost to follow-up did not differ significantly between groups. Interestingly, mortality was significantly lower in the group of patients discharged on dabigatran in comparison to those discharged on warfarin and rivaroxaban. However, we noted that the patient cohort prescribed dabigatran at discharge was on average younger, had less comorbidities, and a higher rate of prior independence. Hence, we interpret this difference in mortality as a selection effect with the patient cohort prescribed dabigatran being considerably younger and healthier.

Patients on dabigatran showed a noticeably weaker adherence, albeit this finding did not reach statistical significance. There are several possible reasons for the relatively high switching rate of $\sim 30 \%$. One reason, which is often cited as a factor impeding medication adherence, is the twice daily (bid) dosing regimen. A meta-analysis of clinical trials with electrical dose-taking monitoring revealed that dose-taking compliance declined as the number of daily doses increased. In the referred meta-analysis, the compliance for od regimens was $80 \%$ and $70 \%$ for bid regimens. ${ }^{18}$ Since apixaban, another NOAC requiring bid dosing, was not yet available during the recruitment period of our registry, we cannot prove a substance-independent negative effect of bid dosing on OAT adherence. The other two reasons given by patients who switched from dabigatran (impairment of renal function and gastrointestinal adverse events) lie within the known profile of this drug. From our point of view, this should not dissuade stroke neurologists from prescribing dabigatran, especially in view of the recently published US Food and Drug Administration drug safety analysis of dabigatran in daily practice. This analysis confirmed the superior risk-to-benefit profile in comparison to VKA based on a study on more than 134,000 Medicare beneficiaries and 37,500 person-years of follow-up, now even in terms of survival. ${ }^{19}$ It may, however, be worthwhile to put special emphasis on the significance of adherence in dabigatran-treated patients.

VKA and rivaroxaban both showed good adherence rates of over $80 \%$, with the limitation that rivaroxaban was only entering the European market during the enrollment period (EU approval of dabigatran September 2011, rivaroxaban December 2011, and apixaban November 2012) and only 35 patients discharged on rivaroxaban were available for follow-up. Among these, only four had switched treatment and none of the patients indicated a specific reason. Approximately $15 \%$ of warfarin-anticoagulated patients switched treatment, mostly to novel NOAC, and the most common reasons to switch (labile INRs and the NOACs greater ease of use) were also expected from the profile of this drug class.

The high adherence to the general principle of any OAT observed in our cohort supports the assumption that the importance of OAT in the secondary prevention of cardioembolic stroke is well-recognized among patients and physicians alike. This fits well with the assumption that prior stroke, which represents a dramatic live event, is a factor independently associated with better adherence to long-term OAT after multivariate analysis for other influencing factors. ${ }^{4}$ One limitation in this context is the fact that we do not have a control group with a less severe condition requiring OAT. Our data were collected from patients treated for stroke at three high-volume academic hospitals in Germany, so questions could be raised as to their generalizability to other medical systems. While an anticoagulation rate at discharge of $55 \%$ appears astonishingly low, it is similar but still somewhat higher than that of a study from the Get With The GuidelinesStroke program of the American Heart Association published in $2011^{20}(45 \%)$. So far, guidelines do not provide substantiated recommendations on when to safely resume OAT after a stroke. In the acute phase, an increased risk of intracranial hemorrhage fostered by the recent ischemic brain injury has to be weighed against the risk of a recurrent embolic stroke. 
However, the fact that only $64 \%$ of our registry's patients discharged without an OAT were put on any OAT at 1-year follow-up (data not shown) should prompt stroke neurologists to reconsider the time point to resume OAT after a stroke. Rather, strong suggestions should be made to install an OAT during the rehabilitation phase or stroke patients should be even scheduled for an outpatient visit to resume OAT. A very recent single center study ${ }^{21}$ describes a patient cohort with stroke and AF of whom a substantially higher percentage (70\%) were discharged on OAT. Even though the authors did not provide data on the length of stay (stroke to OAT interval) and did not systematically screen for complications associated with early installment of OAT, their data imply that it may be safe and effective to install OAT earlier than currently practised ${ }^{20}$ and that stroke centers have an important impact of the implementation of OAT for secondary stroke prevention. As supported by experimental findings, ${ }^{22}$ NOAC may be the safer alternative to VKA for early initiation of VKA. Our data and the aforementioned single center study also show a slightly better 1-year adherence to VKA than a study from the US based on insurance data. ${ }^{4}$ But we would attribute these differences rather to a growing attention toward oral anticoagulation for secondary stroke prevention than toward regional differences.

\section{Conclusion}

Our data, with the limitation of a relatively small but wellselected sample of patients with oral anticoagulation installed for secondary stroke prevention, show that overall adherence to OAT is strong $(>90 \%)$. In our cohort of 324 patients with AF followed-up for 12 months after the initiation of OAT in the course of an ischemic stroke, we did not find a significant positive or negative effect of the specific choice of OAT (VKA or NOAC) on medication adherence. There were relevant - but in our sample not statistically significant - differences in the switching rates of the od-dosed VKA and rivaroxaban on the one hand and the bid-dosed dabigatran on the other hand, but reassuringly, patients who switched treatment mostly switched to another OAT. We conclude that NOAC, even if they do not warrant regular "adherence checks" via mandatory coagulation monitoring, do not have a negative impact on medication adherence but rather enhance OAT use by providing treatment alternatives according to individual tolerability and resources. We therefore speculate that the possibility to exchange one OAT against another might lead to a better overall anticoagulation rate in patients with an indication for OAT as secondary stroke prevention.

\section{Acknowledgment}

The MMAS-8 questionnaire was obtained from Prof Donald E Morisky. Use of the CMMAS is protected by US copyright laws. Permission for use is required. A license agreement is available from: Donald E Morisky, ScD, ScM, MSPH, Professor, Department of Community Health Sciences, UCLA School of Public Health, 650 Charles E Young Drive South, Los Angeles, CA 90095-1772, USA. The authors received no specific funding for this work.

\section{Disclosure}

Dr Kraft has received a travel grant from Bayer Healthcare. Professor Neumann-Haefelin has received speaker's honoraria from Boehringer Ingelheim and Bayer Healthcare. Professor Kleinschnitz has received personal compensation from Bayer Healthcare, Boehringer Ingelheim and BMS/ Pfizer. Professor Steinmetz has received personal compensation from Bayer Healthcare and Boehringer Ingelheim. Dr Foerch received a research grant from Boehringer Ingelheim for investigating dabigatran in an experimental model of traumatic brain injury. The other authors report no conflicts of interest in this work.

\section{References}

1. No authors listed. Secondary prevention in non-rheumatic atrial fibrillation after transient ischaemic attack or minor stroke. EAFT (European Atrial Fibrillation Trial) Study Group. Lancet. 1993; 342(8882):1255-1262.

2. No authors listed. Warfarin versus aspirin for prevention of thromboembolism in atrial fibrillation: Stroke Prevention in Atrial Fibrillation II Study. Lancet. 1994;343(8899):687-691.

3. Haynes RB, Yao X, Degani A, Kripalani S, Garg A, McDonald HP Interventions to enhance medication adherence. Cochrane Database Syst Rev. 2005;4:CD000011.

4. Fang MC, Go AS, Chang Y, et al. Warfarin discontinuation after starting warfarin for atrial fibrillation. Circ Cardiovasc Qual Outcomes. 2010; 3(6):624-631.

5. Ewen S, Rettig-Ewen V, Mahfoud F, Böhm M, Laufs U. Drug adherence in patients taking oral anticoagulation therapy. Clin Res Cardiol. 2014;103(3):173-182

6. Connolly SJ, Ezekowitz MD, Yusuf S, Eikelboom J, Oldgren J, Parekh A, et al. Dabigatran versus warfarin in patients with atrial fibrillation. N Engl J Med. 2009;361(19):1139-1151.

7. Patel MR, Mahaffey KW, Garg J, et al. Rivaroxaban versus warfarin in nonvalvular atrial fibrillation. $N$ Engl J Med. 2011;365(10): $883-891$.

8. Granger CB, Alexander JH, McMurray JJ, et al. Apixaban versus warfarin in patients with atrial fibrillation. $N$ Engl J Med. 2011;365(11): 981-992.

9. Giugliano RP, Ruff CT, Braunwald E, et al. Edoxaban versus warfarin in patients with atrial fibrillation. $N$ Engl J Med. 2013;369(22): 2093-2104.

10. Liew A, Eikelboom JW, O’Donnell M. Randomized controlled trials of new oral anticoagulants for stroke prevention in atrial fibrillation. Curr Opin Cardiol. 2012;27(4):331-339.

11. Dantas GC, Thrompson BV, Manson JA, Tracy CS, Upshur RE. Patients' perspectives on taking warfarin: qualitative study in family practice. BMC Fam Pract. 2004;5:15. 
12. Kernan WN, Ovbiagele B, Black HR, et al. Guidelines for the prevention of stroke in patients with stroke and transient ischemic attack: a guideline for healthcare professionals from the American Heart Association/ American Stroke Association. Stroke. 2014;45(7):2160-2236.

13. Luger S, Hohmann C, Kraft P, et al. Prescription frequency and predictors for the use of novel direct oral anticoagulants for secondary stroke prevention in the first year after their marketing in Europe - a multicentric evaluation. Int J Stroke. 2014;9(5):569-575.

14. Morisky DE, Ang A, Krousel-Wood M, Ward H. Predictive validity of a medication adherence measure for hypertension control. J Clin Hypertens. 2008;10(5):348-354.

15. Morisky DE, DiMatteo MR. Improving the measurement of selfreported medication nonadherence: final response. J Clin Epidemiol. 2011;64(3):262-263.

16. Krousel-Wood M, Islam T, Webber LS, Re RN, Morisky DE, Muntner P. New medication adherence scale versus pharmacy fill rates in seniors with hypertension. Am J Manag Care. 2009;15(1):59-66.

17. Wang Y, Kong MC, Ko Y. Psychometric properties of the 8-item Morisky Medication Adherence Scale in patients taking warfarin. Thromb Haemost. 2012;108(4):789-795.
18. Claxton AJ, Cramer J, Pierce C. A systematic review of the association between dose regimens and medication compliance. Clin Ther. 2001; 23(8):1296-1310.

19. US Food and Drug Administration. FDA drug safety communication 05/13/2014 on Pradaxa (dabigatran). Silver Spring, MD: US Food and Drug Administration. Available from: http://www.fda.gov/safety/ medwatch/safetyinformation/safetyalertsforhumanmedicalproducts/ ucm397179.htm. Accessed April 10, 2015.

20. Lewis WR, Fonarow GC, Grau-Sepulveda MV, et al. Improvement in use of anticoagulation therapy in patients with ischemic stroke: results from Get With The Guidelines-Stroke. Am Heart J. 2011; 162(4):692-699.

21. Sauer R, Sauer EM, Bobinger T, et al. Adherence to oral anticoagulation in secondary stroke prevention - the first year of direct oral anticoagulants. J Stroke Cerebrovasc Dis. 2015;24(1):78-82.

22. Gliem M, Hermsen D, van Rooijen N, Hartung HP, Jander S. Secondary intracerebral hemorrhage due to early initiation of oral anticoagulation after ischemic stroke: an experimental study in mice. Stroke. 2012; 43(12):3352-3357. 


\section{Supplementary materials}

Table SI Demographic characterization by specific choice of OAT

\begin{tabular}{llll}
\hline & VKA & Dabigatran & Rivaroxaban \\
\hline Age (years) Mean \pm SD & $77.4 \pm 8.0$ & $74.2 \pm 10.4$ & $78.2 \pm 8.6$ \\
Female sex & $49.0 \%$ & $48.6 \%$ & $57.1 \%$ \\
Hypertension & $94.9 \%$ & $91.9 \%$ & $96.4 \%$ \\
Diabetes mellitus & $28.7 \%$ & $18.0 \%$ & $33.9 \%$ \\
Prior independence & $79.6 \%$ & $83.8 \%$ & $78.6 \%$ \\
Prior warfarin use & $64.3 \%$ & $11.7 \%$, $* * * *, ., *$ & $30.4 \%$ \\
Serum creatinine level & $1.29 \pm 0.9 \mathrm{mg} / \mathrm{dL}$ & $0.9 \pm 0.3 \mathrm{mg} / \mathrm{dL}$ *.* & $1.0 \pm 0.4 \mathrm{mg} / \mathrm{dL}$ \\
Mean \pm SD & $114.0 \pm 80.0 \mu \mathrm{mol} / \mathrm{L}$ & $79.6 \pm 27 \mu \mathrm{mol} / \mathrm{L}$ & $88.4 \pm 35.4 \mu \mathrm{mol} / \mathrm{L}$ \\
\hline
\end{tabular}

Notes: Patient characteristics at discharge. ${ }^{*}$ In comparison to VKA; ${ }^{\text {in }}$ comparison to rivaroxaban. $* P<0.05$; $* * * P<0.001$ after Bonferroni correction.

Abbreviations: OAT, oral anticoagulant therapy; SD, standard deviation; VKA, vitamin $\mathrm{K}$ antagonists.

Table S2 Reasons to switch therapy during the I-year follow-up period

\begin{tabular}{|c|c|c|c|}
\hline & $\begin{array}{l}\text { Change from VKA } \\
\text { to other treatment } \\
n=14 \text { of } 85 *(16.4 \%)\end{array}$ & $\begin{array}{l}\text { Change from dabigatran } \\
\text { to other treatment } \\
n=22 \text { of } 76 *(28.9 \%)\end{array}$ & $\begin{array}{l}\text { Change from rivaroxaban } \\
\text { to other treatment } \\
n=4 \text { of } 35 *(11.4 \%)\end{array}$ \\
\hline Physician's concerns & Four patients & Eight patients & One patient \\
\hline Patient's concerns & - & - & - \\
\hline Adverse events & Three patients & Eight patients & One patient \\
\hline Costs of therapy & - & One patient & One patient \\
\hline Other & Three patients & Eight patients & One patient \\
\hline Reasons for medication change as & "Easier use of DOAC" $(2 \times)$ & "Impaired renal function" $(2 \times)$ & "Not well tolerated" \\
\hline \multirow[t]{6}{*}{ detailed by patient or caregiver } & "Anemia attributed to VKA" & "Bleeding from gastric ulcers" $(2 \times)$ & \\
\hline & “Labile INR” $(4 \times)$ & "Dyspeptic symptoms" $(2 \times)$ & \\
\hline & "VKA suspected as precipitating & "Easier use of a once daily regime" $(2 \times)$ & \\
\hline & factor for psychotic episode" & "Skin changes attributed to dabigatran" & \\
\hline & & "Vertigo attributed to dabigatran" & \\
\hline & & "Family doctor: no experience with dabigatran" & \\
\hline
\end{tabular}

Notes: *Patients available at I-year follow-up. Patients could provide more than one reason in the questionnaire.

Abbreviations: DOAC, direct oral anticoagulants; INR, international normalized ratio; VKA, vitamin K antagonists.

\section{Publish your work in this journal}

Patient Preference and Adherence is an international, peer-reviewed, open access journal that focuses on the growing importance of patient preference and adherence throughout the therapeutic continuum. Patient satisfaction, acceptability, quality of life, compliance, persistence and their role in developing new therapeutic modalities and compounds to optimize
Dovepress

clinical outcomes for existing disease states are major areas of interest for the journal. This journal has been accepted for indexing on PubMed Central. The manuscript management system is completely online and includes a very quick and fair peer-review system, which is all easy to use. Visit http://www. dovepress.com/testimonials.php to read real quotes from published authors. 